\title{
Minimum Weight $H$-Decompositions of Graphs: The Bipartite Case
}

\author{
Teresa Sousa * \\ Departamento de Matemática and CMA-UNL \\ Faculdade de Ciências e Tecnologia, Universidade Nova de Lisboa \\ Quinta da Torre, 2829-516 Caparica, Portugal \\ tmjs@fct.unl.pt
}

Submitted: Jan 17, 2011; Accepted: May 24, 2011; Published: Jun 6, 2011

Mathematics Subject Classification: 05C70

\begin{abstract}
Given graphs $G$ and $H$ and a positive number $b$, a weighted $(H, b)$-decomposition of $G$ is a partition of the edge set of $G$ such that each part is either a single edge or forms an $H$-subgraph. We assign a weight of $b$ to each $H$-subgraph in the decomposition and a weight of 1 to single edges. The total weight of the decomposition is the sum of the weights of all elements in the decomposition. Let $\phi(n, H, b)$ be the the smallest number such that any graph $G$ of order $n$ admits an $(H, b)$-decomposition with weight at most $\phi(n, H, b)$. The value of the function $\phi(n, H, b)$ when $b=1$ was determined, for large $n$, by Pikhurko and Sousa [Minimum H-Decompositions of Graphs, Journal of Combinatorial Theory, B, 97 (2007), 1041-1055.] Here we determine the asymptotic value of $\phi(n, H, b)$ for any fixed bipartite graph $H$ and any value of $b$ as $n$ tends to infinity.
\end{abstract}

\section{Introduction}

Let $G$ and $H$ be two graphs and $b$ a positive number. A weighted $(H, b)$-decomposition of $G$ is a partition of the edge set of $G$ such that each part is either a single edge or forms an $H$-subgraph, i.e., a graph isomorphic to $H$. We allow partitions only, that is, every edge of $G$ appears in precisely one part. We assign a weight of $b$ to each $H$-subgraph in the decomposition and a weight of 1 to single edges. The total weight of the decomposition is the sum of the weights of all elements in the decomposition. Let $\phi(G, H, b)$ be the smallest possible weight in an $(H, b)$-decomposition of $G$.

\footnotetext{
${ }^{*}$ This work was partially supported by Financiamento Base 2011 ISFL-1-297 and PTDC/MAT/113207/2009 from FCT/MCTES/PT
} 
Let $e(H)$ denote the number of edges in the graph $H$. If $b \geq e(H)$ we have $\phi(G, H, b)=$ $e(G)$. In the case when $0<b<e(H)$ and $H$ is a fixed graph we can easily see that $\phi(G, H, b)=e(G)-p_{H}(G)(e(H)-b)$, where $p_{H}(G)$ is the maximum number of pairwise edge-disjoint $H$-subgraphs that can be packed into $G$. Building upon a body of previous research, Dor and Tarsi [5] showed that if $H$ has a component with at least 3 edges then the problem of checking whether an input graph $G$ admits a partition into $H$-subgraphs is NP-complete. Thus, it is NP-hard to compute the function $\phi(G, H, b)$ for such $H$.

Our goal is to study the function

$$
\phi(n, H, b)=\max \{\phi(G, H, b) \mid v(G)=n\},
$$

which is the smallest number such that any graph $G$ with $n$ vertices admits an $(H, b)$ decomposition with weight at most $\phi(n, H, b)$.

Pikhurko and Sousa [11] considered the case $b=1$ and proved the following results for large $n$.

Theorem 1.1. Let $H$ be any fixed graph of chromatic number $r \geq 3$. Then,

$$
\phi(n, H, 1)=t_{r-1}(n)+o\left(n^{2}\right),
$$

where $t_{r}(n)$, called the Túran number, is the maximum number of edges of an r-partite graph on $n$ vertices.

For a non-empty graph $H$, let $\operatorname{gcd}(H)$ denote the greatest common divisor of the degrees of $H$. For example, $\operatorname{gcd}\left(K_{6,4}\right)=2$ while for any tree $T$ with at least 2 vertices we have $\operatorname{gcd}(T)=1$.

Theorem 1.2. Let $H$ be a bipartite graph with $m$ edges and let $d=\operatorname{gcd}(H)$. Then there is $n_{0}=n_{0}(H)$ such that for all $n \geq n_{0}$ the following statements hold.

If $d=1$, then if $\left(\begin{array}{l}n \\ 2\end{array}\right) \equiv m-1(\bmod m)$,

$$
\phi(n, H, 1)=\phi\left(n, K_{n}, 1\right)=\left\lfloor\frac{n(n-1)}{2 m}\right\rfloor+m-1,
$$

otherwise,

$$
\phi(n, H, 1)=\phi\left(n, K_{n}^{*}, 1\right)=\left\lfloor\frac{n(n-1)}{2 m}\right\rfloor+m-2
$$

where $K_{n}^{*}$ denotes any graph obtained from $K_{n}$ after deleting at most $m-1$ edges in order to have $e\left(K_{n}^{*}\right) \equiv m-1(\bmod m)$. Furthermore, if $G$ is extremal then $G$ is either $K_{n}$ or $K_{n}^{*}$.

If $d \geq 2$, then

$$
\phi(n, H, 1)=\frac{n d}{2 m}\left(\left\lfloor\frac{n}{d}\right\rfloor-1\right)+\frac{1}{2} n(d-1)+O(1) .
$$

Moreover, there is a procedure with running time polynomial in $\log n$ which determines $\phi(n, H, 1)$ and describes a family $\mathcal{D}$ of $n$-sequences such that a graph $G$ of order $n$ satisfies $\phi(G, H, 1)=\phi(n, H, 1)$ if and only if the degree sequence of $G$ belongs to $\mathcal{D}$. (It will be the case that $|\mathcal{D}|=O(1)$ and each sequence in $\mathcal{D}$ has $n-O(1)$ equal entries, so $\mathcal{D}$ can be described using $O(\log n)$ bits.) 
Our goal in this paper is to find the value of the function $\phi(n, H, b)$ for any fixed bipartite graph $H$ and $b \neq 1$.

\section{The bipartite case}

Let $H$ be any fixed bipartite graph. We start this section with an easy Lemma.

Lemma 2.1. Let $H$ be a bipartite graph with $m$ edges and let $b \geq m$ be a constant. Then,

$$
\phi(n, H, b)=\left(\begin{array}{l}
n \\
2
\end{array}\right) .
$$

Proof. Since $b \geq m=e(H)$, we clearly have $\phi(n, G, b)=e(G) \leq\left(\begin{array}{l}n \\ 2\end{array}\right)$ for all graphs $G$ of order $n$. Therefore $\phi(n, H, b) \leq\left(\begin{array}{l}n \\ 2\end{array}\right)$. To prove the lower bound observe that $\phi\left(n, K_{n}, b\right) \geq$ $\frac{b}{m}\left(\begin{array}{l}n \\ 2\end{array}\right) \geq\left(\begin{array}{l}n \\ 2\end{array}\right)$.

Recall that for a non-empty $\operatorname{graph} H, \operatorname{gcd}(H)$ denotes the greatest common divisor of the degrees of $H$. We will prove the following result.

Theorem 2.2. Let $H$ be a bipartite graph with $m$ edges, let $d=\operatorname{gcd}(H)$ and $0<b<m$ with $b \neq 1$ a constant. Then there is $n_{0}=n_{0}(H)$ such that for all $n \geq n_{0}$ the following statements hold.

If $d=1$, then

$$
\phi(n, H, b)=b \frac{n(n-1)}{2 m}+O(1) .
$$

If $d \geq 2$, let $n-1=q d+r$ where $0 \leq r \leq d-1$ is an integer.

If $r \neq 0$ and $d-1 \leq \frac{b d}{m}+r$, then

$$
\phi(n, H, b)=\frac{b}{m}\left(\begin{array}{l}
n \\
2
\end{array}\right)+\frac{1}{2} n\left(r-\frac{b r}{m}\right)+O(1) .
$$

If $r \neq 0$ and $d-1 \geq \frac{b d}{m}+r$, then

$$
\phi(n, H, b)=\frac{b}{m}\left(\begin{array}{l}
n \\
2
\end{array}\right)+\frac{1}{2} n\left(d-1-\frac{b r}{m}-\frac{b d}{m}\right)+O(1) .
$$

If $r=0$ and $\frac{b}{m}<1-\frac{5 d^{2}}{5 d^{3}-2}$, then

$$
\phi(n, H, b)=\frac{b}{m}\left(\begin{array}{l}
n \\
2
\end{array}\right)+\frac{1}{2} n\left(d-1-\frac{b d}{m}\right)+O(1) .
$$

If $r=0$ and $1-\frac{5 d^{2}}{5 d^{3}-2} \leq \frac{b}{m} \leq 1-\frac{1}{d}$, then

$$
\frac{b}{m}\left(\begin{array}{l}
n \\
2
\end{array}\right)+\frac{1}{2} n\left(d-1-\frac{b d}{m}\right)-\frac{1}{2} \leq \phi(n, H, b) \leq \frac{b}{m}\left(\begin{array}{l}
n \\
2
\end{array}\right)+\frac{m-b}{5 m d^{2}} n .
$$




$$
\begin{aligned}
& \text { If } r=0 \text { and } \frac{b}{m} \geq 1-\frac{1}{d} \text {, then } \\
& \qquad \frac{b}{m}\left(\begin{array}{l}
n \\
2
\end{array}\right) \leq \phi(n, H, b) \leq \frac{b}{m}\left(\begin{array}{l}
n \\
2
\end{array}\right)+\frac{m-b}{5 m d^{2}} n .
\end{aligned}
$$

Before we start the proof, we provide some auxiliary results. We start with the following result appearing in Pikhurko and Sousa [11, Theorem 3.1].

Lemma 2.3. For any bipartite graph $H$ with bipartition $\left(V_{1}, V_{2}\right)$ and any $A \subset V_{1}$ with $a \geq 1$ elements, there are integers $C$ and $n_{0}$ such that the following holds. In any graph $G$ of order $n \geq n_{0}$ with minimum degree $\delta(G) \geq \frac{2}{3} n$ there is a family of edge disjoint copies of $H$ such that the vertex subsets corresponding to $A \subset V(H)$ are disjoint and cover all but at most $C$ vertices of $G$. One can additionally ensure that each vertex of $G$ belongs to at most $3(v(H))^{2}$ copies of $H$.

The following results appearing in Alon, Caro and Yuster [1, Theorem 1.1, Corollary 3.4, Lemma 3.5] which follow with some extra work from the powerful decomposition theorem of Gustavsson [8], are crucial to the proof of our result.

Lemma 2.4. For any non-empty graph $H$ with $m$ edges, there are $\gamma>0$ and $N_{0}$ such that the following holds. Let $d=\operatorname{gcd}(H)$. Let $G$ be a graph of order $n \geq N_{0}$ and of minimum degree $\delta(G) \geq(1-\gamma) n$.

If $d=1$, then

$$
p_{H}(G)=\left\lfloor\frac{e(G)}{m}\right\rfloor
$$

If $d \geq 2$, let $\alpha_{u}=d\left\lfloor\frac{\operatorname{deg}(u)}{d}\right\rfloor$ for $u \in V(G)$ and let $X$ consist of all vertices whose degree is not divisible by $d$. If $|X| \geq \frac{n}{10 d^{3}}$, then

$$
p_{H}(G)=\left\lfloor\frac{1}{2 m} \sum_{u \in V(G)} \alpha_{u}\right\rfloor .
$$

$$
\text { If }|X|<\frac{n}{10 d^{3}} \text {, then }
$$

$$
p_{H}(G) \geq \frac{1}{m}\left(e(G)-\frac{n}{5 d^{2}}\right)
$$

Proof of Theorem 2.2. Given $H$, let $\gamma(H)$ and $N_{0}$ be given by Lemma 2.4. Assume that $\gamma \leq \gamma(H)$ is sufficiently small and that $n_{0} \geq N_{0}$ is sufficiently large to satisfy all the inequalities we will encounter. Let $n \geq n_{0}$ and let $G$ be any graph of order $n$ with $\phi(G, H, b)=\phi(n, H, b)$. We will follow the proof of Pikhurko and Sousa [11, Theorem 1.4], thus only the main results will be stated.

Let $G_{n}=G$. Repeat the following at most $\lfloor n / \log n\rfloor$ times: If the current graph $G_{i}$ has a vertex $x_{i}$ of degree at most $(1-\gamma / 2) i$, let $G_{i-1}=G_{i}-x_{i}$ and decrease $i$ by 1 . Suppose we stopped after $s$ repetitions. Pikhurko and Sousa proved that $s<\lfloor n / \log n\rfloor$ and the graph $G_{n-s}$ has $\delta\left(G_{n-s}\right) \geq(1-\gamma / 2)(n-s)$. 
Let $\alpha=2 \gamma$. We will have another pass over the vertices $x_{n}, \ldots, x_{n-s+1}$, each time decomposing the edges incident to $x_{i}$ by $H$-subgraphs and single edges. It will be the case that each time we remove the edges incident to the current vertex $x_{i}$, the degree of any other vertex drops by at most $3 h^{4}$, where $h=v(H)$. Here is a formal description. Initially, let $G_{n}^{\prime}=G$ and $i=n$. If in the current graph $G_{i}^{\prime}$ we have $\operatorname{deg}_{G_{i}^{\prime}}\left(x_{i}\right) \leq \alpha n$, then we remove all $G_{i}^{\prime}$-edges incident to $x_{i}$ as single edges and let $G_{i-1}^{\prime}=G_{i}^{\prime}-x_{i}$.

Suppose that $\operatorname{deg}_{G_{i}^{\prime}}\left(x_{i}\right)>\alpha n$. Then, the set $X_{i}=\left\{y \in V\left(G_{n-s}\right): x_{i} y \in E\left(G_{i}^{\prime}\right)\right\}$, has at least $\alpha n-s+1$ vertices. The minimum degree of $G\left[X_{i}\right]$ is

$$
\delta\left(G\left[X_{i}\right]\right) \geq\left|X_{i}\right|-s-\frac{\gamma n}{2}-s \times 3 h^{4} \geq \frac{2}{3}\left|X_{i}\right| .
$$

Let $y \in V(H), A=N_{H}(y)$ and $a=|A|$. By Lemma 2.3 there is a constant $C$ such that all but at most $C$ vertices of $G\left[X_{i}\right]$ can be covered by edge disjoint copies of $H-y$ each of them having vertex disjoint sets $A$. Therefore, all but at most $C$ edges between $x_{i}$ and $X_{i}$ can be decomposed into copies of $H$. All other edges incident to $x_{i}$ are removed as single edges. Let $G_{i-1}^{\prime}$ consist of the remaining edges of $G_{i}^{\prime}-x_{i}$ (that is, those edges that do not belong to an $H$-subgraph of the above $x_{i}$-decomposition). This finishes the description of the case $\operatorname{deg}_{G_{i}^{\prime}}\left(x_{i}\right)>\alpha n$.

Consider the sets $S={ }^{i}\left\{x_{n}, \ldots, x_{n-s+1}\right\}, S_{1}=\left\{x_{i} \in S: \operatorname{deg}_{G_{i}^{\prime}}\left(x_{i}\right) \leq \alpha n\right\}$, and $S_{2}=S \backslash S_{1}$. Let their sizes be $s, s_{1}$ and $s_{2}$ respectively, so $s=s_{1}+s_{2}$.

Let $F$ be the graph with vertex set $V\left(G_{n-s}\right) \cup S_{2}$, consisting of the edges coming from the removed $H$-subgraphs when we processed the vertices in $S_{2}$. We have

$$
\phi(G, H, b) \leq \phi\left(G_{n-s}^{\prime}, H, b\right)+b \frac{e(F)}{m}+s_{1} \alpha n+s_{2} C+\left(\begin{array}{l}
s \\
2
\end{array}\right) .
$$

We know that $\phi\left(G_{n-s}^{\prime}, H, b\right)=e\left(G_{n-s}^{\prime}\right)-p_{H}\left(G_{n-s}^{\prime}\right)(m-b)$. The last statement of Lemma 2.3 guarantees that $\delta\left(G_{n-s}^{\prime}\right) \geq(1-\gamma)(n-s)$. Thus, $p_{H}\left(G_{n-s}^{\prime}\right)$ can be estimated using Lemma 2.4.

Consider first the case $d=1$. Using the inequalities $e(F) \leq(1-\gamma / 2) s_{2} n$ and $\alpha \leq$ $b(2-\gamma) / 2 m$, we obtain

$$
\begin{aligned}
\phi(G, H, b) & \leq \phi\left(G_{n-s}^{\prime}, H, b\right)+b \frac{e(F)}{m}+s_{1} \alpha n+s_{2} C+\left(\begin{array}{l}
s \\
2
\end{array}\right) \\
& \leq e\left(G_{n-s}^{\prime}\right)-p_{H}\left(G_{n-s}^{\prime}\right)(m-b)+b \frac{e(F)}{m}+s_{1} \alpha n+s_{2} C+\left(\begin{array}{l}
s \\
2
\end{array}\right) \\
& \leq e\left(G_{n-s}^{\prime}\right)-\left\lfloor\frac{e\left(G_{n-s}^{\prime}\right)}{m}\right\rfloor(m-b)+b \frac{e(F)}{m}+s_{1} \alpha n+s_{2} C+\left(\begin{array}{l}
s \\
2
\end{array}\right) \\
& \leq\left(\frac{b}{m}\left(\begin{array}{c}
n-s \\
2
\end{array}\right)+m-b\right)+b \frac{2-\gamma}{2 m} s_{2} n+b \frac{2-\gamma}{2 m} s_{1} n+s_{2} C+\left(\begin{array}{l}
s \\
2
\end{array}\right) \\
& \leq \frac{b}{m}\left(\begin{array}{c}
n-s \\
2
\end{array}\right)+b \frac{2-\gamma}{2 m} s n+s_{2} C+\left(\begin{array}{l}
s \\
2
\end{array}\right)+m-b \\
& \leq \frac{b}{m}\left(\begin{array}{l}
n \\
2
\end{array}\right)-b \frac{(n-1) s}{m}+b \frac{s(s-1)}{2 m}+b \frac{2-\gamma}{2 m} s n+s_{2} C+\left(\begin{array}{l}
s \\
2
\end{array}\right)+m-b .
\end{aligned}
$$


If $S \neq \emptyset$ then in order to prove that $\phi(G, H, b)<\frac{b}{m}\left(\begin{array}{c}n \\ 2\end{array}\right) \leq \phi\left(H, K_{n}, b\right)$ and hence a contradiction to our assumption on $G$, it suffices to show that

$$
b \frac{s}{m}+b \frac{s(s-1)}{2 m}+\left(\begin{array}{l}
s \\
2
\end{array}\right)+s_{2} C+m-b<\left(\frac{b}{m}-\frac{b(2-\gamma)}{2 m}\right) n s .
$$

But this last inequality holds since we have $s<\frac{n}{\log n}$ and $n$ is sufficiently large. Thus, $S=\emptyset$ and

$$
\begin{aligned}
\phi(G, H, b) & =e(G)-(m-b)\left\lfloor\frac{e(G)}{m}\right\rfloor \\
& \leq b \frac{e(G)}{m}+(m-b) \\
& \leq b \frac{n(n-1)}{2 m}+(m-b),
\end{aligned}
$$

giving us the upper bound. To prove the lower bound we consider the complete graph on $n$ vertices and we obtain

$$
\phi\left(K_{n}, H, b\right)=e\left(K_{n}\right)-(m-b)\left\lfloor\frac{e\left(K_{n}\right)}{m}\right\rfloor \geq b \frac{n(n-1)}{2 m} .
$$

Consider the case $d \geq 2$ and let $n-1=q d+r$ with $0 \leq r \leq d-1$ an integer. To prove the lower bounds we consider the complete graph of order $n \geq n_{0}$ and a graph $L$ of order $n \geq n_{0}$, which is (almost) ( $q d-1$ )-regular (except at most one vertex of degree $q d-2$ ). (Such a graph $L$ exists, which can be seen either directly or from Erdős and Gallai's result [6].) We have,

$$
\begin{aligned}
\phi\left(K_{n}, H, b\right) & =e\left(K_{n}\right)-p_{H}\left(K_{n}\right)(m-b) \\
& \geq\left(\begin{array}{l}
n \\
2
\end{array}\right)-\frac{1}{2}-\frac{n d q}{2 m}(m-b) \\
& \geq \frac{b}{m}\left(\begin{array}{l}
n \\
2
\end{array}\right)+\frac{1}{2} n\left(r-\frac{b r}{m}\right)-\frac{1}{2},
\end{aligned}
$$

and,

$$
\begin{aligned}
\phi(L, H, b) & =e(L)-p_{H}(L)(m-b) \\
& \geq \frac{1}{2} n(q d-1)-\frac{1}{2}-\frac{n d(q-1)}{2 m}(m-b) \\
& \geq \frac{b}{m}\left(\begin{array}{l}
n \\
2
\end{array}\right)+\frac{1}{2} n\left(d-1-\frac{b r}{m}-\frac{b d}{m}\right)-\frac{1}{2},
\end{aligned}
$$

giving the required lower bounds in view of $q=\frac{n-1-r}{d}$.

We will now prove the upper bounds. 
Assume first that (2.9) holds. Then, by (2.10)

$$
\begin{aligned}
\phi(G, & H, b) \\
& \leq \phi\left(G_{n-s}^{\prime}, H, b\right)+b \frac{e(F)}{m}+s_{1} \alpha n+s_{2} C+\left(\begin{array}{l}
s \\
2
\end{array}\right) \\
& \leq e\left(G_{n-s}^{\prime}\right)-p_{H}\left(G_{n-s}^{\prime}\right)(m-b)+b \frac{e(F)}{m}+s_{1} \alpha n+s_{2} C+\left(\begin{array}{l}
s \\
2
\end{array}\right) \\
& \leq e\left(G_{n-s}^{\prime}\right)-\frac{m-b}{m}\left(e\left(G_{n-s}^{\prime}\right)-\frac{n-s}{5 d^{2}}\right)+b \frac{e(F)}{m}+s_{1} \alpha n+s_{2} C+\left(\begin{array}{l}
s \\
2
\end{array}\right) \\
& \leq \frac{b}{m}\left(\begin{array}{c}
n-s \\
2
\end{array}\right)+\frac{b(2-\gamma)}{2 m} s_{2} n+\frac{(m-b)(n-s)}{5 m d^{2}}+\frac{b(2-\gamma)}{2 m} s_{1} n+s_{2} C+\left(\begin{array}{l}
s \\
2
\end{array}\right) \\
& \leq \frac{b}{m}\left(\begin{array}{l}
n \\
2
\end{array}\right)-\frac{b(n-1) s}{m}+\frac{b s(s-1)}{2 m}+\frac{(m-b)(n-s)}{5 m d^{2}}+\frac{b(2-\gamma)}{2 m} s n+s_{2} C+\left(\begin{array}{l}
s \\
2
\end{array}\right) .
\end{aligned}
$$

For $s>\frac{2(m-b)}{5 \gamma d^{2} b}$ we have $\frac{b}{m}-\frac{b(2-\gamma)}{2 m}-\frac{m-b}{5 m d^{2} s}>0$. Thus, for $n$ sufficiently large

$$
\frac{b s}{m}+\frac{b s(s-1)}{2 m}-\frac{(m-b) s}{5 m d^{2}}+s_{2} C+\left(\begin{array}{l}
s \\
2
\end{array}\right)<\left(\frac{b}{m}-\frac{b(2-\gamma)}{2 m}-\frac{m-b}{5 m d^{2} s}\right) n s .
$$

That is, $\phi(G, H, b)<\frac{b}{m}\left(\begin{array}{l}n \\ 2\end{array}\right) \leq \phi\left(K_{n}, H, b\right)$ which contradicts the optimality of $G$. Otherwise, $s$ is bounded by a constant independent of $n$ and the coefficient of $s n$ is $-\frac{b}{m}+\frac{b(2-\gamma)}{2 m}<0$. Thus, for the case $r \neq 0$, to obtain the contradiction $\phi(G, H, b)<$ $\phi\left(K_{n}, H, b\right)$ it suffices to show that

$$
\frac{b}{m}\left(\begin{array}{l}
n \\
2
\end{array}\right)+\frac{m-b}{5 m d^{2}} n<\frac{b}{m}\left(\begin{array}{l}
n \\
2
\end{array}\right)+\frac{1}{2} n\left(r-\frac{b r}{m}\right)
$$

that is,

$$
\frac{1}{5 d^{2}}<\frac{1}{2} r
$$

which holds since $d \geq 2$ and $r \geq 1$.

If $r=0$ and $\frac{b}{m}<1-\frac{5 d^{2}}{5 d^{3}-2}$, to obtain the contradiction $\phi(G, H, b)<\phi(L, H, b)$ it suffices to show that

$$
\frac{b}{m}\left(\begin{array}{l}
n \\
2
\end{array}\right)+\frac{m-b}{5 m d^{2}} n<\frac{b}{m}\left(\begin{array}{l}
n \\
2
\end{array}\right)+\frac{1}{2} n\left(d-1-\frac{b d}{m}\right),
$$

which holds since $\frac{b}{m}<1-\frac{5 d^{2}}{5 d^{3}-2}$. Otherwise, we have

$$
\phi(G, H, b)<\frac{b}{m}\left(\begin{array}{l}
n \\
2
\end{array}\right)+\frac{m-b}{5 m d^{2}} n
$$

which is the upper bound stated in (2.5) and (2.6). 
Finally, assume that (2.8) holds. It follows that $p_{H}(G)$ and thus $\phi(G, H, b)$, depends only on the degree sequence $d_{1}, \ldots, d_{n}$ of $G$. Namely, the packing number $\ell=p_{H}(G)$ equals $\left\lfloor\frac{1}{2 m} \sum_{i=1}^{n} r_{i}\right\rfloor$, where $r_{i}=d\left\lfloor d_{i} / d\right\rfloor$ is the largest multiple of $d$ not exceeding $d_{i}$.

Thus, it is enough for us to prove the upper bounds in (2.3) and (2.4) on $\phi_{\max }$, the maximum of

$$
\phi\left(d_{1}, \ldots, d_{n}\right)=\frac{1}{2} \sum_{i=1}^{n} d_{i}-(m-b)\left\lfloor\frac{1}{2 m} \sum_{i=1}^{n}\left\lfloor\frac{d_{i}}{d}\right\rfloor d\right\rfloor,
$$

over all (not necessarily graphical) sequences $d_{1}, \ldots, d_{n}$ of integers with $0 \leq d_{i} \leq n-1$.

Let $d_{1}, \ldots, d_{n}$ be an optimal sequence attaining the value $\phi_{\max }$. For $i=1, \ldots, n$ let $d_{i}=q_{i} d+r_{i}$ with $0 \leq r_{i} \leq d-1$. Then, $\ell=\left\lfloor\frac{\left(q_{1}+\cdots+q_{n}\right) d}{2 m}\right\rfloor$.

Recall that $n-1=q d+r$ with $1 \leq r \leq d-1$. Define $R=q d-1$ to be the maximum integer which is at most $n-1$ and is congruent to $d-1$ modulo $d$. Let $C_{1}=\left\{i \in[n]: r_{i}=d-1\right.$ and $\left.d_{i}<R\right\}$ and $C_{2}=\left\{i \in[n]: d_{i}=n-1\right\}$ if $n-1 \neq R$ and $C_{2}=\emptyset$ otherwise.

Since $d_{1}, \ldots, d_{n}$ is an optimal sequence, we have that if $r_{i} \neq d-1$ then $d_{i}=n-1$ for all $i \in[n]$. Also, $\left|C_{1}\right| \leq \frac{2 m}{d}-1$ and $\left|C_{2}\right| \leq 2 m-1$. We have

$$
\begin{aligned}
\frac{1}{2} \sum_{i=1}^{n} d_{i} & =\frac{1}{2}\left(n-\left|C_{1} \cup C_{2}\right|\right) R+\frac{1}{2} \sum_{i \in C_{1}} d_{i}+\frac{1}{2}\left|C_{2}\right|(n-1) \\
& \leq \frac{1}{2} n d(q-1)+\frac{1}{2} n(d-1)-\frac{d}{2} \sum_{i \in C_{1}}\left(q-1-q_{i}\right)+O(1), \\
\ell & \geq\left(\frac{1}{2 m} \sum_{i=1}^{n}\left\lfloor\frac{d_{i}}{d}\right\rfloor d\right)-1 \\
& \geq \frac{1}{2 m} n d(q-1)-\frac{d}{2 m} \sum_{i \in C_{1}}\left(q-1-q_{i}\right)+O(1) .
\end{aligned}
$$

These estimates give us the required upper bound in (2.3) and (2.4).

$$
\begin{aligned}
\phi_{\max }=\frac{1}{2} \sum_{i=1}^{n} d_{i}-(m-b) \ell & \leq \frac{b}{2 m} n d(q-1)+\frac{1}{2} n(d-1)+O(1) \\
& \leq \frac{b}{m}\left(\begin{array}{c}
n \\
2
\end{array}\right)+\frac{1}{2} n\left(d-1-\frac{b r}{m}-\frac{b d}{m}\right)+O(1) .
\end{aligned}
$$

The upper bound in (2.2) follows from the fact that

$$
\frac{b}{m}\left(\begin{array}{l}
n \\
2
\end{array}\right)+\frac{1}{2} n\left(d-1-\frac{b r}{m}-\frac{b d}{m}\right) \leq \frac{b}{m}\left(\begin{array}{l}
n \\
2
\end{array}\right)+\frac{1}{2} n\left(r-\frac{b r}{m}\right),
$$

in view of $d-1 \leq \frac{b d}{m}+r$. 
To finish the proof it remains to obtain a contradiction if $S \neq \emptyset$ holds. Let $\bar{d}_{1}, \ldots, \bar{d}_{n}$ be the degree sequence of the graph with vertex set $V(G)$ and edge set $E\left(G_{n-s}^{\prime}\right) \cup E(F)$. Consider the new sequence of integers

$$
d_{i}^{\prime}= \begin{cases}\bar{d}_{i}, & \text { if } x_{i} \notin S, \\ \bar{d}_{i}+\left\lceil\frac{(1-3 \gamma)}{m} n\right\rceil m, & \text { if } x_{i} \in S_{1}, \\ \bar{d}_{i}+\left\lceil\frac{\gamma}{4 m} n\right\rceil m, & \text { if } x_{i} \in S_{2} .\end{cases}
$$

Each $d_{i}^{\prime}$ lies between 0 and $n-1$, so $\phi\left(d_{1}^{\prime}, \ldots, d_{n}^{\prime}\right) \leq \phi_{\max }$. We obtain

$$
\begin{aligned}
\phi(G, H, b) & \leq \phi\left(\bar{d}_{1}, \ldots, \bar{d}_{n}\right)+s_{1} \alpha n+s_{2} C+\left(\begin{array}{l}
s \\
2
\end{array}\right) \\
& <\phi\left(d_{1}^{\prime}, \ldots, d_{n}^{\prime}\right)-\frac{b(1-3 \gamma)}{2 m} s_{1} n-\frac{b \gamma}{8 m} s_{2} n+s_{1} \alpha n+s_{2} C+\left(\begin{array}{l}
s \\
2
\end{array}\right) \\
& <\phi\left(d_{1}^{\prime}, \ldots, d_{n}^{\prime}\right)-\left(\frac{b(1-3 \gamma)}{2 m}-2 \gamma\right) s_{1} n-\frac{b \gamma}{8 m} s_{2} n+s_{2} C+\left(\begin{array}{l}
s \\
2
\end{array}\right) \\
& <\phi\left(d_{1}^{\prime}, \ldots, d_{n}^{\prime}\right)-\frac{b \gamma}{8 m} s_{1} n-\frac{b \gamma}{8 m} s_{2} n+s_{2} C+\left(\begin{array}{l}
s \\
2
\end{array}\right) \\
& \leq \phi_{\max }-\frac{b \gamma}{10 m} s n,
\end{aligned}
$$

which contradicts the already established facts that $\phi(n, H, b)$ is at most $\phi(G, H, b)$ by the optimality of $G$ and is at least $\phi_{\max }$ by (2.16).

Acknowledgement. The author thanks Oleg Pikhurko for helpful discussions and comments.

\section{References}

[1] N. Alon, Y. Caro, and R. Yuster, Packing and covering dense graphs, J. Combin. Designs 6 (1998), 451-472.

[2] N. Alon and R. Yuster, H-factors in dense graphs, J. Combin. Theory (B) 66 (1996), 269-282.

[3] B. Bollobás, On complete subgraphs of different orders, Math. Proc. Camb. Phil. Soc. 79 (1976), 19-24.

[4] H. Chernoff, A measure of asymptotic efficiency for tests of a hypothesis based on the sum of observations., Ann. Math. Statistics 23 (1952), 493-507.

[5] D. Dor and M. Tarsi, Graph decomposition is NP-complete: a complete proof of Holyer's conjecture, SIAM J. Computing 26 (1997), 1166-1187.

[6] P. Erdős and T. Gallai, Graphs with prescribed degree of vertices, Mat. Lapok 11 (1960), 264-274. 
[7] P. Erdős, A. W. Goodman, and L. Pósa, The representation of a graph by set intersections, Can. J. Math. 18 (1966), 106-112.

[8] T. Gustavsson, Decompositions of large graphs and digraphs with high minimum degree, Ph.D. thesis, Univ. of Stockholm, 1991.

[9] J. Komlós, G. N. Sárkőzy, and E. Szemerédi, Proof of the Alon-Yuster conjecture, Discrete Math. 235 (2001), 255-269.

[10] P. Kővari, V. T. Sös, and P. Turán, On a problem of K. Zarankiewicz, Colloq. Math. 3 (1954), 50-57.

[11] Pikhurko, Oleg and Sousa, Teresa, Minimum H-Decompositions of Graphs, Journal of Combinatorial Theory, B, 97 (2007), 1041-1055.

[12] N. Pippenger and J. Spencer, Asymtotic behavior of the chromatic index for hypergraphs, J. Combin. Theory (A) 51 (1989), 24-42.

[13] V. Rödl, On a packing and covering problem, Europ. J. Combin. 5 (1985), 69-78.

[14] A. Shokoufandeh and Y. Zhao, Proof of a tiling conjecture of Komlós, Random Struct. Algorithms 23 (2003), 180-205.

[15] M. Simonovits and V. T. Sós, Szemerédi's partition and quasirandomness, Random Struct. Algorithms 2 (1991), 1-10.

[16] T. Sousa, Decompositions of graphs into 5-cycles and other small graphs, Electronic J. Combin. 12 (2005), 7pp.

[17] T. Sousa, Decompositions of graphs into a given clique-extension, Submitted, 2005.

[18] E. Szemerédi, Regular partitions of graphs, Proc. Colloq. Int. CNRS, Paris, 1976, pp. 309-401. 